\author{
А.В. Андреев \\ Объединенный институт высоких температур РАН, Москва, Россия \\ СУПЕРПОЗИЦИЯ СТЕПЕННО-ЛОГАРИФМИЧЕСКИХ \\ И СТЕПЕННЫХ СИНГУЛЯРНЫХ РЕШЕНИЙ В ДВУМЕРНЫХ \\ ЗАДАЧАХ ТЕОРИИ УПРУГОСТИ
}

\begin{abstract}
Выполнено сопоставление полученных ранее результатов для степенной и степенно-логарифмической сингулярной асимптотики решения одного класса сингулярных интегральных уравнений плоской теории упругости. Отмечается, что для указанных типов сингулярных решений характеристические части интегрального уравнения содержат подобные члены. В связи с этим построены трансцендентные уравнения относительно показателей особенности аддитивной формы (суперпозиции) степенной и степенно-логарифмической асимптотик. Показано, что такая суперпозиция сингулярных решений реализуется с показателем особенности, известным для классической степенной асимптотики упругих напряжений.

Обсуждается общий характер этого результата, связанный с описанием многочисленных краевых задач плоской теории упругости системами сингулярных интегральных уравнений, принадлежащих рассматриваемому классу. На основе теории комплексных потенциалов КолосоваМусхелишвили построено сингулярное степенно-логарифмическое решение одной краевой задачи, иллюстрирующее полученные результаты с позиций методов прямого асимптотического анализа краевых задач теории упругости.

Предложен подход к параметризации уравнений относительно вещественного показателя особенности, позволяющий расширить область реализации неосциллирующего сингулярного решения. Представлены результаты расчетов показателя главной степенно-логарифмической особенности для задачи о трещине, достигающей границы раздела материалов. Продемонстрирована эффективность разработанного параметрического подхода на примере такой задачи.

Ключевые слова: сингулярное интегральное уравнение, степенная и степенно-логарифмическая асимптотика, концентрация упругих напряжений, комплексный и вещественный показатель особенности.
\end{abstract}

\title{
A.V. Andreev
}

Joint Institute for High Temperatures RAS, Moscow, Russian Federation

\section{SUPERPOSITION OF POWER-LOGARITHMIC AND POWER SINGULAR SOLUTIONS IN TWO-DIMENSIONAL ELASTICITY PROBLEMS}

A comparison of the results obtained recently for power and power-logarithmic singular asymptotics of solution associated with a class of singular integral equations of the two-dimensional elasticity is performed. It is noted that leading parts of the integral equation contain similar terms for these singu- 
lar solutions. In this connection, transcendental equations in regard to singularity exponents for additive form (superposition) of power and power-logarithmic solution asymptotics were constructed. It was established that superposition of the mentioned singular solutions has the singularity exponent which is known for the classical power asymptotics of elastic stress.

The general nature of the obtained results is discussed that is related to the description of numerous boundary value problems of the two-dimensional elasticity by means of systems of singular integral equations belonging to the class under consideration. Based on theory of the KolosovMuskhelishvili complex potentials power-logarithmic singular solution of a boundary value problem is constructed. This solution represents the obtained results from point of view of direct asymptotic analysis of the boundary value problems.

The parametric approach for equations on real singularity exponent is suggested to extend domain where non-oscillatory asymptotic is implemented. Numerical results on leading power-logarithmic singularity exponent for the two-dimensional problem of the elasticity theory for the crack terminating an interface are presented. The efficiency of the developed parametric approach for examined crack problem is demonstrated.

Keywords: singular integral equation, power and power-logarithmic asymptotics, elastic stress concentration, complex and real singularity exponent.

\section{Введение}

Разрушение конструкций и природных объектов сопровождается, как правило, ростом трещин или трещиноподобных дефектов, которые являются наиболее опасными концентраторами напряжений. Кроме того, потеря несущей способности элементами конструкций и функциональные отказы деталей машин зачастую обусловлены развитием пластической деформации из областей концентрации напряжений. Особое место в классификации различных типов концентраторов занимают угловые (конические) точки кусочно-гладкой границы тела, поскольку вблизи таких особых точек упругие напряжения могут возрастать неограниченно, т.е. оказываются сингулярными. Аналогичными концентраторами внутри упругого тела являются точки нарушения гладкости на линии (поверхности), где имеет место существенная локальная неоднородность материала. Сингулярные упругие решения также может порождать изменение типа краевых условий, которое свойственно, например, контактным задачам. В связи с актуальностью проблемы анализа сингулярных упругих решений проводились многочисленные прямые исследования конкретных краевых задач при различных их геометрических конфигурациях, механических параметрах и граничных условиях в окрестности особых точек [1-3]. В данной работе анализ сингулярных решений плоской теории упругости выполнен с позиций метода сингулярных интегральных уравнений $[4,5]$, который позволил получить весьма общие и важные новые результаты. 


\section{1. Предварительные результаты}

В работах [4, 5] рассматривалось сингулярное интегральное уравнение (СИУ)

$$
\begin{gathered}
A(\eta) \varphi(\eta)+B(\eta) \int_{-1}^{1} \frac{\varphi(\xi) d \xi}{\xi-\eta}+\int_{-1}^{1} K_{g e n}(\xi, \eta) \varphi(\xi) d \xi+\int_{-1}^{1} L_{g e n}(\xi, \eta) \overline{\varphi(\xi)} d \xi+ \\
+\int_{-1}^{1} K(\xi, \eta) \varphi(\xi) d \xi+\int_{-1}^{1} L(\xi, \eta) \overline{\varphi(\xi)} d \xi=p(\eta),-1<\eta<1,
\end{gathered}
$$

где $\varphi(\xi)$ и $\overline{\varphi(\xi)}$ - соответственно неизвестная и комплексно сопряженная с ней функция; $p(\eta)$ - заданная на отрезке $[-1,1]$ функция; функции $p(\eta), A(\eta)$ и $B(\eta)$ ограничены при $\eta \rightarrow \pm 1$, причем $A(\eta)$ и $B(\eta)$ имеют пределы при $\eta \rightarrow \pm 1$; ядра $K(\xi, \eta)$ и $L(\xi, \eta)$ удовлетворяют условию Гельдера по обеим переменным. Полагалось $[4,5]$, что ядра $K_{g e n}(\xi, \eta)$ и $L_{g e n}(\xi, \eta)$ являются обобщенными ядрами типа Коши [6] $\left(K_{\text {gen }}(\xi, \eta), L_{g e n}(\xi, \eta) \sim 1 / x, x \rightarrow 0\right.$ при $\left.\xi=\eta \rightarrow \pm 1\right)$ и представимы в форме

$$
\begin{gathered}
K_{g e n}(\xi, \eta)=\sum_{p, j, k, r}^{\prime} a_{k}(\eta) \frac{(1+\xi)^{p}(1+\eta)^{j}}{\left(\xi-z_{r}^{*}\right)^{p+j+1}}+\sum_{l, m, n, s}^{\prime} b_{l}(\eta) \frac{(1-\xi)^{m}(1-\eta)^{n}}{\left(\xi-z_{s}^{* *}\right)^{m+n+1}} \\
L_{g e n}(\xi, \eta)=\sum_{p, j, k, r}^{\prime \prime} c_{k}(\eta) \frac{(1+\xi)^{p}(1+\eta)^{j}}{\left(\xi-z_{r}^{*}\right)^{p+j+1}}+\sum_{l, m, n, s}^{\prime \prime} d_{l}(\eta) \frac{(1-\xi)^{m}(1-\eta)^{n}}{\left(\xi-z_{s}^{* *}\right)^{m+n+1}} \\
z_{r}^{*}(\eta)=-1+(1+\eta) e^{i \theta_{r}}, 0<\theta_{r}<2 \pi ; z_{s}^{* *}(\eta)=1+(1-\eta) e^{i \theta_{s}},-\pi<\theta_{s}<\pi ; i^{2}=-1 .
\end{gathered}
$$

Здесь индексы и показатели степени $p, j, k, l, m, n, r$, $s=0,1,2, \ldots$ изменяются независимо в рамках каждой из сумм, а заданные функции $a_{k}(\eta), b_{l}(\eta), c_{k}(\eta)$ и $d_{l}(\eta)$ ограничены и имеют пределы при $\eta \rightarrow \pm 1$.

В [4] был разработан метод определения особенностей степенного типа в решении для СИУ (1) с обобщенными ядрами (2) и (3), при этом использовалось представление для главной части решения в форме

$$
\varphi(\xi)=u(\xi)(1-\xi)^{\alpha}(1+\xi)^{\beta},-1<\operatorname{Re} \alpha, \operatorname{Re} \beta<0,
$$

где $\alpha$ и $\beta$ - отыскиваемые показатели особенности, в общем случае комплексные, а $u(\xi)$ - комплексная функция, ограниченная на концах 
промежутка интегрирования, где она не обращается в нуль и имеет соответствующие пределы $u( \pm 1)$. На основе асимптотического анализа интегралов СИУ (1) было показано [4], что для формы решения (4) характеристические части СИУ при $\eta \rightarrow \pm 1$ представимы в виде

$$
\begin{aligned}
& I_{\text {sing }}^{-}(\eta)=\left.2^{\alpha} \Delta_{1}^{-}(\beta)\left\{(1+\eta)^{\beta}\right\}\right|_{\eta \rightarrow-1} u(-1)+\left.2^{\bar{\alpha}} \Delta_{2}^{-}(\bar{\beta})\left\{(1+\eta)^{\bar{\beta}}\right\}\right|_{\eta \rightarrow-1} \overline{u(-1)}, \\
& I_{\text {sing }}^{+}(\eta)=\left.2^{\beta} \Delta_{1}^{+}(\alpha)\left\{(1-\eta)^{\alpha}\right\}\right|_{\eta \rightarrow+1} u(+1)+\left.2^{\bar{\beta}} \Delta_{2}^{+}(\bar{\alpha})\left\{(1-\eta)^{\bar{\alpha}}\right\}\right|_{\eta \rightarrow+1} \overline{u(+1)} .
\end{aligned}
$$

Здесь ограниченные функции $\Delta_{1}^{-}(\beta), \Delta_{2}^{-}(\bar{\beta}), \Delta_{1}^{+}(\alpha)$ и $\Delta_{2}^{+}(\bar{\alpha})$ определяются параметрами СИУ (1) и обобщенных ядер (2) и (3), выражения для них приведены в $[4,5]$. Исходя из условия ограниченности характеристических частей (5) и (6) на концах промежутка интегрирования были построены следующие уравнения для комплексных

$$
\begin{aligned}
& \Delta_{1}^{-}(\beta) \overline{\Delta_{1}^{-}(\beta)}-\Delta_{2}^{-}(\bar{\beta}) \overline{\Delta_{2}^{-}(\bar{\beta})}=0, \\
& \Delta_{1}^{+}(\alpha) \overline{\Delta_{1}^{+}(\alpha)}-\Delta_{2}^{+}(\bar{\alpha}) \overline{\Delta_{2}^{+}(\bar{\alpha})}=0
\end{aligned}
$$

и вещественных

$$
\begin{aligned}
& \Delta_{1}^{-}(\beta) \overline{\Delta_{1}^{-}}(\beta)-\Delta_{2}^{-}(\beta) \overline{\Delta_{2}^{-}}(\beta)=0 \\
& \Delta_{1}^{+}(\alpha) \overline{\Delta_{1}^{+}}(\alpha)-\Delta_{2}^{+}(\alpha) \overline{\Delta_{2}^{+}}(\alpha)=0
\end{aligned}
$$

показателей степенной особенности решения [4]. Анализ этих уравнений, в частности, выявил, что распределение комплексных корней носит непрерывный характер, т.е. уравнения (7) и (8) выделяют бесконечное множество допустимых для СИУ (1) комплексных показателей степенной особенности решения, в то время как вещественные корни (9) и (10), как правило, составляют дискретный конечный набор [4]. Оба этих свойства вытекают непосредственно из формы уравнений (7)-(10), левые части которых в силу взаимной сопряженности множителей являются вещественными. Следовательно, уравнения (7) и (8) накладывают ограничение только на взаимосвязь вещественной и мнимой частей показателя особенности. Отметим, что такой нетривиальный характер показателей особенности обусловлен наличием сопряженной функции в характеристической части СИУ (1), а при ее отсутствии на данном конце промежутка интегрирования из (5) и (6) следуют уравнения 


$$
\begin{aligned}
& \Delta_{1}^{-}(\beta)=0, \\
& \Delta_{1}^{+}(\alpha)=0,
\end{aligned}
$$

которые предоставляют дискретные, как правило, наборы допустимых для СИУ (1) показателей степенной особенности. Во всех случаях, включая непрерывное распределение корней, главный показатель степенной особенности соответствует корню с минимальной вещественной частью в полосе $-1<\operatorname{Re} \alpha, \operatorname{Re} \beta<0$.

В работе [5] было выполнено аналогичное исследование для особенностей степенно-логарифмического типа на основе асимптотической формы решения СИУ (1):

$$
\varphi(\xi)=\varphi_{+1}(1-\xi)^{\alpha} \ln (1-\xi)+\varphi_{-1}(1+\xi)^{\beta} \ln (1+\xi)+\varphi_{0}(\xi),-1<\operatorname{Re} \alpha, \operatorname{Re} \beta<0 .
$$

Здесь $\varphi_{+1}$ и $\varphi_{-1}-$ некоторые отличные от нуля комплексные постоянные, а функция $\varphi_{0}(\xi)$ обращается в нуль на концах промежутка интегрирования. Характеристическая часть СИУ (1) при $\eta \rightarrow-1$ в этом случае имеет вид [5]

$$
\begin{gathered}
I_{\text {sing }}^{-}(\eta)=\left.\Delta_{1}^{-}(\beta) \varphi_{-1}\left\{(1+\eta)^{\beta} \ln (1+\eta)\right\}\right|_{\eta \rightarrow-1}+\left.\Delta_{2}^{-}(\bar{\beta}) \overline{\varphi_{-1}}\left\{(1+\eta)^{\bar{\beta}} \ln (1+\eta)\right\}\right|_{\eta \rightarrow-1}+ \\
+\left.\Delta_{3}^{-}(\beta) \varphi_{-1}\left\{(1+\eta)^{\beta}\right\}\right|_{\eta \rightarrow-1}+\left.\Delta_{4}^{-}(\bar{\beta}) \overline{\varphi_{-1}}\left\{(1+\eta)^{\bar{\beta}}\right\}\right|_{\eta \rightarrow-1},
\end{gathered}
$$

где ограниченные функции $\Delta_{3}^{-}(\beta), \Delta_{4}^{-}(\bar{\beta})$ также определяются параметрами СИУ (1) и ядер (2) и (3), выражения для них приведены в [5] (там же представлена характеристическая часть СИУ при $\eta \rightarrow+1$ ). Исходя из условия ограниченности характеристической части (14) на концах промежутка интегрирования были построены три различных варианта уравнения относительно показателя степенно-логарифмической особенности $\beta$

$$
\begin{gathered}
\Delta_{1}^{-}(\beta) \Delta_{4}^{-}(\bar{\beta})-\Delta_{2}^{-}(\bar{\beta}) \Delta_{3}^{-}(\beta)=0, \\
\left\{\begin{array}{l}
\left|\Delta_{1}^{-}(\beta)\right|^{2}-\left|\Delta_{2}^{-}(\bar{\beta})\right|^{2}=0, \\
\left|\Delta_{3}^{-}(\beta)\right|^{2}-\left|\Delta_{4}^{-}(\bar{\beta})\right|^{2}=0,
\end{array}\right. \\
\Delta_{1}^{-}(\bar{\beta}) \overline{\Delta_{3}^{-}(\beta)}-\Delta_{2}^{-}(\bar{\beta}) \overline{\Delta_{4}^{-}(\bar{\beta})}=0
\end{gathered}
$$


и аналогичные по структуре уравнения относительно показателя особенности $\alpha[5]$.

Как видно, уравнения (15)-(17) могут предоставить комплексные показатели степенно-логарифмической особенности для асимптотической формы (13) (в [5] они были определены на примере одной прикладной задачи), однако для вещественных показателей особенности уравнения (15)-(17) оказываются переопределенными, и, следовательно, в общем случае сингулярное решение в форме (13) с вещественными показателями не является допустимым для СИУ (1) с обобщенными ядрами (2) и (3). Кроме того, в [5] было показано, что в отсутствие неподвижных особенностей в ядре $L_{g e n}(\xi, \eta)(3)$, например, на левом конце промежутка интегрирования показатель степенно-логарифмической особенности $\beta$ для асимптотической формы решения (13) должен удовлетворять системе уравнений

$$
\left\{\begin{array}{l}
\Delta_{1}^{-}(\beta)=0, \\
\Delta_{3}^{-}(\beta)=0
\end{array}\right.
$$

которая является переопределенной как для вещественных, так уже и для комплексных значений $\beta$. Аналогичная переопределенная система имеет место для показателя степенно-логарифмической особенности $\alpha$ формы (13), когда ядро (3) не имеет неподвижных особенностей на правом конце промежутка интегрирования.

Полученные ранее $[4,5]$ и кратко представленные здесь результаты асимптотического анализа решения СИУ (1) с ядрами в форме (2) и (3) позволяют сделать важный вывод. Как следует из представления (14) для характеристической части СИУ (1), его интегралы, вычисляемые для степенно-логарифмической асимптотики решения (13), порождают как степенно-логарифмические, так и степенные сингулярные члены. Поскольку аналогичные степенные сингулярные члены (см. (5) и (6)) также возникают в характеристической части для классической степенной асимптотики решения (4), вклады степенно-логарифмической и степенной асимптотик в характеристическую часть оказываются подобными, и этот факт указывает на необходимость дальнейшего анализа в следующей постановке. 


\section{2. Показатели особенности суперпозиции сингулярных решений}

Представим главную часть решения СИУ (1) в аддитивной форме, сочетающей степенно-логарифмическую и степенную асимптотики с равными показателями особенности $(-1<\operatorname{Re} \alpha, \operatorname{Re} \beta<0)$ :

$$
\varphi(\xi)=\varphi_{+1}(1-\xi)^{\alpha} \ln (1-\xi)+\varphi_{-1}(1+\xi)^{\beta} \ln (1+\xi)+u(\xi)(1-\xi)^{\alpha}(1+\xi)^{\beta} .
$$

Здесь, как и ранее, $\varphi_{+1}$ и $\varphi_{-1}-$ отличные от нуля комплексные постоянные, а $u(\xi)$ - комплексная функция, которая имеет пределы на концах промежутка интегрирования $u( \pm 1)$, где она ограничена и не обращается в нуль. Как следует из сопоставления (4), (5) и (13), (14), для главной части решения в форме сингулярной суперпозиции (19) характеристическая часть СИУ (1) при $\eta \rightarrow-1$ принимает вид

$$
\begin{gathered}
I_{\text {sing }}^{-}(\eta)=\left.\Delta_{1}^{-}(\beta) \varphi_{-1}\left\{(1+\eta)^{\beta} \ln (1+\eta)\right\}\right|_{\eta \rightarrow-1}+ \\
+\left.\Delta_{2}^{-}(\bar{\beta}) \overline{\varphi_{-1}}\left\{(1+\eta)^{\bar{\beta}} \ln (1+\eta)\right\}\right|_{\eta \rightarrow-1}+ \\
+\left.\left[\Delta_{3}^{-}(\beta) \varphi_{-1}+2^{\alpha} \Delta_{1}^{-}(\beta) u(-1)\right]\left\{(1+\eta)^{\beta}\right\}\right|_{\eta \rightarrow-1}+ \\
+\left.\left[\Delta_{4}^{-}(\bar{\beta}) \overline{\varphi_{-1}}+2^{\bar{\alpha}} \Delta_{2}^{-}(\bar{\beta}) \overline{u(-1)}\right]\left\{(1+\eta)^{\bar{\beta}}\right\}\right|_{\eta \rightarrow-1} .
\end{gathered}
$$

Если вынести здесь за скобки неограниченно возрастающие множители, из условия ограниченности характеристической части СИУ [4-7] при $\eta \rightarrow-1$ приравнять оставшиеся множители к нулю, то получим систему уравнений

$$
\left\{\begin{array}{l}
\left.\Delta_{1}^{-}(\beta) \varphi_{-1}\left\{(1+\eta)^{i \operatorname{Im} \beta}\right\}\right|_{\eta \rightarrow-1}+\left.\Delta_{2}^{-}(\bar{\beta}) \overline{\varphi_{-1}}\left\{(1+\eta)^{-i \operatorname{Im} \beta}\right\}\right|_{\eta \rightarrow-1}=0, \\
{\left.\left[\Delta_{3}^{-}(\beta) \varphi_{-1}+2^{\alpha} \Delta_{1}^{-}(\beta) u(-1)\right]\left\{(1+\eta)^{i \operatorname{Im} \beta}\right\}\right|_{\eta \rightarrow-1}+} \\
+\left.\left[\Delta_{4}^{-}(\bar{\beta}) \overline{\varphi_{-1}}+2^{\bar{\alpha}} \Delta_{2}^{-}(\bar{\beta}) \overline{u(-1)}\right]\left\{(1+\eta)^{-i \operatorname{Im} \beta}\right\}\right|_{\eta \rightarrow-1}=0 .
\end{array}\right.
$$

Присоединив к ним сопряженные уравнения, можно сформировать систему четырех однородных уравнений относительно значений $\varphi_{-1} \overline{\varphi_{-1}}, u(-1)$ и $\overline{u(-1)}$. Приравнивая к нулю определитель этой системы, сокращая одинаковые множители и раскрывая неопределенности, 
после упрощений получим уравнение относительно комплексного показателя особенности $\beta$ в сингулярной суперпозиции (19)

$$
\Delta_{1}^{-}(\beta) \overline{\Delta_{1}^{-}(\beta)}-\Delta_{2}^{-}(\bar{\beta}) \overline{\Delta_{2}^{-}(\bar{\beta})}=0 .
$$

Как видно, это уравнение в точности совпадает с уравнением (7), имеющим место для степенной, без логарифмических множителей, асимптотики решения (4). Аналогичным образом можно показать, что относительно комплексного показателя $\alpha$ в сингулярной суперпозиции (19) справедливо уравнение

$$
\Delta_{1}^{+}(\alpha) \overline{\Delta_{1}^{+}(\alpha)}-\Delta_{2}^{+}(\bar{\alpha}) \overline{\Delta_{2}^{+}(\bar{\alpha})}=0
$$

совпадающее с (8). Соответственно, относительно априори вещественных $\alpha$ и $\beta$ имеем уравнения (9) и (10), которые, в отличие от свойственных форме (13) уравнений (15)-(17) (см. также [4, 5]), могут предоставлять вещественные показатели главной степенно-логарифмической особенности в составе сингулярной суперпозиции (19).

Обсудим этот весьма важный для приложений результат, имеющий весьма общий характер, поскольку никаких дополнительных условий при его получении, помимо нетривиальности значений $\varphi_{-1} \varphi_{+1}$, $u(-1)$ и $u(+1)$ в представлении главной части решения в форме сингулярной суперпозиции (19), не накладывалось. Прежде всего заметим, что подобно ситуации для главной части решения в форме (13) в отсутствие сопряженной функции в характеристической части СИУ при $\eta \rightarrow-1$ из соотношений (21) для сингулярной суперпозиции (19) вновь имеем переопределенную систему (18) относительно показателя особенности $\beta$. Аналогичная система определяет показатель особенности $\alpha$ в случае ограниченности ядра (3) при $\eta \rightarrow+1$. Следовательно, если обобщенное ядро $L_{g e n}(\xi, \eta)$ не имеет неподвижных особенностей на данном конце промежутка интегрирования, в общем случае решение СИУ (1) не содержит здесь степенно-логарифмических особенностей. Показатели степенной особенности, реализующейся в рамках такой ситуации, определяются в соответствии с уравнениями (11) и (12). Как отмечалось в [5], этот специальный случай с точки зрения двумерных краевых задач теории упругости соответствует особой точке, вблизи которой задача разделяется на независимые нормальную и сдвиговую 
задачи, или, другими словами, компоненты упругих перемещений определяются независимо друг от друга. В терминах СИУ, описывающего такую краевую задачу, последнее означает, что СИУ (1) распадается на два независимых уравнения относительно двух вещественных неизвестных функций. Как свидетельствуют результаты данного исследования, во всех прочих случаях главная сингулярная асимптотика упругого поля является не степенной, а степенно-логарифмической, причем показатели главной степенно-логарифмической особенности могут предоставляться в том числе уравнениями, соответствующими степенной асимптотике.

Как обсуждалось в [5], анализ степенных особенностей решения СИУ с обобщенными ядрами, описывающего некоторую краевую задачу, ранее был ограничен случаем $L_{g e n}(\xi, \eta) \equiv 0[6-9]$, за исключением работы [4], где рассматривались степенные особенности при $L_{g e n}(\xi, \eta) \neq 0$ (см. также некоторые другие работы, упомянутые в [4]). Были выполнены, однако, многочисленные прямые исследования двумерных краевых задач о сингулярной асимптотике упругого поля напряжений в окрестности его особых точек при различных геометрических и механических параметрах задачи, условиях нагружения и граничных условиях. Использовавшийся подход к асимптотическому анализу был предложен в [10] и получил очень широкое распространение при решении различных задач теории упругости, механики разрушения и контактной механики, связанных с определением особенностей поля напряжений. Этот подход основан на отыскании бигармонической функции напряжений Эйри в специальной форме, отражающей в полярных координатах только главный член степенной асимптотики поля напряжений вблизи особой точки [10]. Удовлетворение граничным условиям, реализующимся вблизи этой точки при различных величинах полярного угла, дает возможность построить характеристическое уравнение относительно показателя особенности поля напряжений. Как обсуждалось в [11], такой подход к построению характеристического уравнения эквивалентен отысканию комплексных потенциалов Колосова-Мусхелишвили [12] в специальной форме, также отражающей только главный член степенной асимптотики решения с вещественным показателем особенности, и последующему удовлетворению граничным условиям в комплексной форме. В дальнейшем техника 
комплексных потенциалов Колосова-Мусхелишвили также широко использовалась для прямого асимптотического анализа краевых задач, было также предложено ее обобщение на случай комплексного показателя степенной асимптотики [13]. Отметим, что аналогичные асимптотические исследования двумерных краевых задач теории упругости проводились также с использованием преобразования Меллина [3], причем в указанной работе обсуждалась также эквивалентность всех трех методик. Обзоры работ данного направления представлены в [1-3]. Существенно, что во всех этих исследованиях в качестве базовой выбиралась степенная асимтотика упругого поля и отыскивались, соответственно, показатели степенной особенности, вещественные либо комплексные. Исключение здесь составляет только небольшой круг работ, обзор которых представлен в [14] (см. также некоторые близкие исследования, упомянутые в [1-3]), где исследовалась степеннологарифмическая асимптотика упругого решения в области перехода от пары вещественных показателей степенной особенности к комплексному показателю. При этом указанными выше средствами асимтотического анализа краевой задачи вопрос определения вещественного показателя степенно-логарифмической особенности сводился к решению характеристической системы уравнений, эквивалентной уравнениям (15)-(17). Поскольку такого рода система для вещественного показателя особенности оказывается переопределенной, далее в пространстве геометрических и механических параметров задачи определялись поверхности (соотношения в виде равенств между этими параметрами), где имеет место вещественная степенно-логарифмическая особенность и показатель этой особенности [14]. Напротив, результаты данного пункта свидетельствуют о том, что любой степенной особенности упругого поля, свойственной той или иной краевой задаче, можно поставить в соответствие более сильную, степенно-логарифмическую особенность, также удовлетворяющую этой краевой задаче. Исключение составляет только обсуждавшийся выше специальный случай, соответствующий высокому уровню симметрии краевой задачи, разделяющейся на независимые нормальную и сдвиговую задачи.

Действительно, двумерные задачи линейной теории упругости для однородных и кусочно-однородных областей, тел с трещинами, вырезами и включениями сводятся к комплексному СИУ, заданному на кусочно-гладком криволинейном контуре, либо, в общем случае, к сис- 
теме таких уравнений (см., например, $[15,16])$. Каждое уравнение такой системы задается на одном контуре, в левой же части СИУ осуществляется суммирование по всем контурам, каждый из которых входит в состав СИУ со своей неизвестной функцией (и сопряженной с ней). В рамках анализа особенности упругого решения вблизи особой точки криволинейные контуры интегрирования в ее окрестности можно без потери общности аппроксимировать прямолинейными, что после канонической параметризации этих контуров приводит к обобщенным ядрам в форме (2) и (3) и сингулярному интегралу указанного в (1) вида. Существенно, что все неизвестные функции, относительно которых записывается такая система СИУ, имеют единую асимптотическую форму вблизи особой точки, что связано с необходимостью удовлетворения тем или иным условиям, описывающим взаимодействие клиновидных областей на их границах. Как следствие, характеристическая часть каждого СИУ системы представляет собой сумму характеристических частей вида тех, которые выписаны выше (см. (5), (14) и (20)), с одинаковыми неограниченно возрастающими множителями. Ограничивая характеристическую часть каждого СИУ и присоединяя к полученным уравнениям сопряженные, получим однородную алгебраическую систему относительно параметров главной части решения (см. также (4), (13) и (19)) вблизи особой точки. Определитель такой алгебраической системы предоставит уравнения относительно показателя степенной или степенно-логарифмической особенности, причем очевидно, что характер этих уравнений и взаимосвязь показателей особенности для форм (4) и (19), выявленные для СИУ (1), будут унаследованы системой СИУ типа (1), определяющих сингулярную асимптотику решения вблизи особой точки.

Рассмотрим ставшую классической задачу Вильямса [10] о сингулярной асимтотике напряжений вблизи вершины упругого однородного клина с углом раствора $2 \gamma(0<\gamma \leq \pi)$. В этом иллюстративном примере ограничимся первой основной задачей с однородными граничными условиями и используем подход [11], базирующийся на технике комплексных потенциалов Колосова-Мусхелишвили [12] как отвечающий используемому в данной работе комплексному формализму и потому наиболее компактный. Запишем комплексные потенциалы в аддитивной форме степенной и степенно-логарифмической асимптотик, соответствующей форме (19): 


$$
\varphi(z)=a z^{\lambda} \ln z+c z^{\lambda}, \psi(z)=b z^{\lambda} \ln z+d z^{\lambda} .
$$

Здесь $a, b, c, d$ - комплексные постоянные, а $\lambda$ - вещественный показатель асимптотики [11], связанный с показателем особенности упругих напряжений $\beta$ соотношением $\lambda=\beta+1(0<\lambda<1)$.

Для первой основной задачи с однородными граничными условиями имеем [12]

$$
\varphi(t)+t \overline{\varphi^{\prime}(t)}+\overline{\psi(t)}=0
$$

где $t$ - переменная точка контура. Удовлетворяя граничные условия (25) в полярных координатах $z=r e^{i \theta}$ на контурах, соответствующих прямолинейным краям клина $\theta=0,2 \gamma$, получим

$$
\begin{gathered}
r^{\lambda} \ln r\left[a e^{i \theta \lambda}+\lambda \bar{a} e^{-i \theta(\lambda-2)}+\bar{b} e^{-i \theta \lambda}\right]+ \\
+r^{\lambda}\left[i \theta a e^{i \theta \lambda}+c e^{i \theta \lambda}-i \theta \lambda \bar{a} e^{-i \theta(\lambda-2)}+(\bar{a}+\lambda \bar{c}) e^{-i \theta(\lambda-2)}-\right. \\
\left.-i \theta \bar{b} e^{-i \theta \lambda}+\bar{d} e^{-i \theta \lambda}\right]=0, \theta=0,2 \gamma .
\end{gathered}
$$

Из условия удовлетворения этих уравнений при любых $r$ имеем систему

$$
\left\{\begin{array}{l}
a+\lambda \bar{a}+\bar{b}=0, \\
a e^{2 i \gamma \lambda}+\lambda \bar{a} e^{-2 i \gamma(\lambda-2)}+\bar{b} e^{-2 i \gamma \lambda}=0, \\
c+\bar{a}+\lambda \bar{c}+\bar{d}=0, \\
2 i \gamma a e^{2 i \gamma \lambda}+c e^{2 i \gamma \lambda}-2 i \gamma \lambda \bar{a} e^{-2 i \gamma(\lambda-2)}+(\bar{a}+\lambda \bar{c}) e^{-2 i \gamma(\lambda-2)}-2 i \gamma \bar{b} e^{-2 i \gamma \lambda}+\bar{d} e^{-2 i \gamma \lambda}=0,
\end{array}\right.
$$

к которой можно присоединить сопряженные уравнения и в такой расширенной системе рассматривать $a, b, c, d$ и $\bar{a}, \bar{b}, \bar{c}, \bar{d}$ как независимые переменные. Существование нетривиального упругого поля $(a, b, c, d \neq 0)$, таким образом, требует обращения в нуль определителя однородной системы из восьми уравнений, откуда после упрощений следует уравнение

$$
\lambda \sin 2 \gamma= \pm \sin (2 \lambda \gamma),
$$

известное для классической степенной асимптотики решения [10], т.е. для потенциалов в форме (24) при $a=b \equiv 0$ [11]. Отметим, что первая основная задача о клине в части определения особенности упругого 
поля в его вершине эквивалентна задаче о двухзвенной ломаной трещине $(0<\vartheta \leq \pi-$ угол между звеньями) в однородном материале [15], которая описывается системой двух СИУ типа (1) при $A(\eta)=0(\forall \vartheta)$ и $L_{g e n}(\xi, \eta) \neq 0$ для всех $0<\vartheta<\pi$ (при $\vartheta=\pi$ излом отсутствует).

Таким образом, данное исследование свидетельствует, что многочисленные результаты [1-3], полученные в рамках анализа поведения решения вблизи особых точек двумерных краевых задач теории упругости, контактной механики и механики разрушения, требуют пересмотра и уточнения.

Определение главной асимптотики упругого поля для уже известных результатов анализа вещественного показателя степенной особенности [1-3] может базироваться на непосредственном учете логарифмического усиления такой особенности в составе суперпозиции сингулярных решений (см. (24)). При этом следует, однако, провести дополнительный качественный анализ конкретной краевой задачи с тем, чтобы исключить обсуждавшийся выше специальный случай, когда это усиление не реализуется. Отметим, что для рассмотренной первой основной задачи Вильямса этот случай соответствует только вырождению особой точки при $\gamma=\pi / 2$ (полуплоскость) и точке возврата контура клина $\gamma=\pi$ (вершина трещины-разреза).

Ситуация с комплексной асимптотикой обстоит несколько сложнее, поскольку комплексные показатели степенно-логарифмической особенности могут предоставляться не только уравнением вида (22) и (23), соответствующим в том числе степенной особенности, но и уравнениями типа (15)-(17), построенными для асимптотической формы (13). Как следствие, главный комплексный показатель степенно-логарифмической особенности в общем случае может не совпадать с комплексным показателем степенной особенности, известным из асимптотического анализа краевой задачи. Методика анализа краевой задачи, соответствующая решению в форме (13) (или, что то же самое, форме (24) при $c=d \equiv 0)$, может быть развита из имеющихся для степенной асимптотики подходов [13], однако такого рода исследование находится за рамками данной статьи.

Отметим также, что данное исследование основано на асимптотическом анализе решения одномерного СИУ, что соответствует двумерной краевой задаче теории упругости (плоское напряженное со- 
стояние или плоская деформация). В то же время полученные результаты непосредственно отражаются на сингулярной асимптотике напряжений в трехмерной задаче о ребре пространственного клина, которая определяется решениями плоской и антиплоской задач [17], а идея суперпозиции степенно-логарифмического и степенного сингулярных решений может найти свое применение и в существенно трехмерных задачах [18, 19].

\section{3. Параметризация уравнений относительно показателя особенности}

Как обсуждалось выше, если для главной части решения в форме (13) уравнения относительно вещественного показателя степеннологарифмической особенности в общем случае оказываются переопределенными (см. (15)-(17)), то для сингулярной суперпозиции (19) относительно вещественного показателя особенности имеют место уравнения (9) и (10), левые части которых являются вещественными. Следовательно, уравнения (9) и (10) могут иметь вещественные корни не только в специальных случаях или с дополнительными ограничениями, накладываемыми на параметры этих уравнений. В то же время при решении конкретных прикладных задач может возникнуть следующая ситуация. Подобно, например, алгебраическому уравнению с вещественными коэффициентами, уравнения (9) и (10) могут иметь в том числе и (попарно сопряженные) комплексные корни. Такие корни, безусловно, следует исключить из рассмотрения, поскольку ни они, ни их вещественные части не удовлетворяют исходному уравнению (7) и (8) и, следовательно, не являются допустимыми для СИУ (1). Отметим, что при асимптотическом анализе краевых задач комплексные корни зачастую рассматриваются наравне с вещественными [1-3], что представляется неправомерным, если при получении характеристического уравнения относительно показателя особенности использовались априорные предположения о его вещественности [11].

Вещественные показатели главной особенности, степенной или степенно-логарифмической, представляют особый интерес в связи с тем, что они, в отличие от комплексных показателей, не порождают осцилляций сингулярных упругих полей вблизи особой точки. В задачах о трещине, например, такого рода осцилляции приводят к перемещениям переменного знака вблизи ее вершины, что в модели трещины- 
разреза соответствует нефизическому взаимопроникновению ее поверхностей. Перестроение математической модели на основе введения области контакта поверхностей трещины, примыкающей к ее вершине, позволяет устранить нефизические эффекты (см. работы, упоминающиеся в [1-3]), однако сохраняет затруднения в интерпретации результатов в части анализа условий роста трещины при ее распространении по механизму нормального отрыва. Полученные в данном исследовании результаты указывают путь к расширению области существования вещественных показателей главной особенности, что при решении прикладных задач позволяет построить сингулярные неосциллирующие решения без паллиативного перестроения математической модели трещины.

Зафиксируем взаимосвязь параметров главной части решения в сингулярной суперпозиции (19) на левом конце промежутка интегрирования соотношением

$$
\lim _{\xi \rightarrow-1} u(\xi)=u(-1)=2^{-\alpha} G^{-} \varphi_{-1}
$$

где $G^{-}$- комплексная постоянная. Тогда систему (21) можно переписать в виде

$$
\left\{\begin{array}{l}
\left.\Delta_{1}^{-}(\beta) \varphi_{-1}\left\{(1+\eta)^{i \operatorname{Im} \beta}\right\}\right|_{\eta \rightarrow-1}+\left.\Delta_{2}^{-}(\bar{\beta}) \overline{\varphi_{-1}}\left\{(1+\eta)^{-i \operatorname{Im} \beta}\right\}\right|_{\eta \rightarrow-1}=0, \\
{\left.\left[\Delta_{3}^{-}(\beta)+G^{-} \Delta_{1}^{-}(\beta)\right] \varphi_{-1}\left\{(1+\eta)^{i \operatorname{Im} \beta}\right\}\right|_{\eta \rightarrow-1}+} \\
+\left.\left[\Delta_{4}^{-}(\bar{\beta})+\overline{G^{-}} \Delta_{2}^{-}(\bar{\beta})\right] \overline{\varphi_{-1}}\left\{(1+\eta)^{-i \operatorname{Im} \beta}\right\}\right|_{\eta \rightarrow-1}=0 .
\end{array}\right.
$$

Как следует из первого уравнения такой системы, второе уравнение не зависит от вещественной части $G^{-}$, поэтому без ограничения общности можно положить $\operatorname{Re} G^{-} \equiv 0$ и обозначить $\operatorname{Im} G^{-} \equiv C^{-}$. Следовательно, соотношение (26) можно переписать в форме

$$
u(-1)=i 2^{-\alpha} C^{-} \varphi_{-1},
$$

где $C^{-}$- вещественный параметр. Подставив (27) в систему (21) и присоединив к ней сопряженные уравнения, из условия равенства нулю определителя подсистемы, состоящей из любой пары однородных уравнений относительно $\varphi_{-1}$ и $\varphi_{+1}$, получим три варианта пара- 
метризованного уравнения относительно комплексного показателя особенности сингулярной суперпозиции (19) в рамках соотношения (27):

$$
\begin{aligned}
& \Delta_{1}^{-}(\beta)\left[\Delta_{4}^{-}(\bar{\beta})-i C^{-} \Delta_{2}^{-}(\bar{\beta})\right]-\Delta_{2}^{-}(\bar{\beta})\left[\Delta_{3}^{-}(\beta)+i C^{-} \Delta_{1}^{-}(\beta)\right]=0, \\
& \left\{\begin{array}{l}
\left|\Delta_{1}^{-}(\beta)\right|^{2}-\left|\Delta_{2}^{-}(\bar{\beta})\right|^{2}=0, \\
\left|\Delta_{3}^{-}(\beta)+i C^{-} \Delta_{1}^{-}(\beta)\right|^{2}-\left|\Delta_{4}^{-}(\bar{\beta})-i C^{-} \Delta_{2}^{-}(\bar{\beta})\right|^{2}=0,
\end{array}\right. \\
& \overline{\Delta_{1}^{-}(\beta)}\left[\Delta_{3}^{-}(\beta)+i C^{-} \Delta_{1}^{-}(\beta)\right]-\overline{\Delta_{2}^{-}(\bar{\beta})}\left[\Delta_{4}^{-}(\bar{\beta})-i C^{-} \Delta_{2}^{-}(\bar{\beta})\right]=0 .
\end{aligned}
$$

Особый интерес такая параметризация представляет для класса вещественных значений показателя $\beta(\operatorname{Im} \beta \equiv 0)$, поскольку параметр $C^{-}$можно рассматривать как дополнительную степень свободы в получаемых для такого случая уравнениях:

$$
\begin{gathered}
\Delta_{1}^{-}(\beta)\left[\Delta_{4}^{-}(\beta)-i C^{-} \Delta_{2}^{-}(\beta)\right]-\Delta_{2}^{-}(\beta)\left[\Delta_{3}^{-}(\beta)+i C^{-} \Delta_{1}^{-}(\beta)\right]=0, \\
\left\{\begin{array}{l}
\left|\Delta_{1}^{-}(\beta)\right|^{2}-\left|\Delta_{2}^{-}(\beta)\right|^{2}=0, \\
\left|\Delta_{3}^{-}(\beta)+i C^{-} \Delta_{1}^{-}(\beta)\right|^{2}-\left|\Delta_{4}^{-}(\beta)-i C^{-} \Delta_{2}^{-}(\beta)\right|^{2}=0,
\end{array}\right. \\
\overline{\Delta_{1}^{-}}(\beta)\left[\Delta_{3}^{-}(\beta)+i C^{-} \Delta_{1}^{-}(\beta)\right]-\overline{\Delta_{2}^{-}}(\beta)\left[\Delta_{4}^{-}(\beta)-i C^{-} \Delta_{2}^{-}(\beta)\right]=0 .
\end{gathered}
$$

Комплексные уравнения (31) и (33) распадаются на системы двух вещественных уравнений, решение которых (как и решение вещественной системы (32)) следует отыскивать относительно двух вещественных переменных - $C^{-}$и $\beta$. Следовательно, уравнения (31)-(33) могут предоставить вещественные главные показатели особенности в тех случаях, когда уравнение (9) не имеет вещественных корней. Следует подчеркнуть, что предлагаемая параметризация решения не накладывает никаких ограничений на параметры СИУ (1) и, как следствие, на механические и геометрические параметры краевой задачи, описываемой этим СИУ.

Аналогичным образом, если для сингулярной суперпозиции (19) на правом конце промежутка интегрирования задать соотношение

$$
\lim _{\xi \rightarrow+1} u(\xi)=u(+1)=i 2^{-\beta} C^{+} \varphi_{+1}
$$


где $C^{+}$- вещественный параметр, можно получить следующие параметризованные уравнения относительно вещественного показателя особенности $\alpha$ (см. также [5]):

$$
\begin{gathered}
\Delta_{1}^{+}(\alpha)\left[\Delta_{4}^{+}(\alpha)-i C^{+} \Delta_{2}^{+}(\alpha)\right]-\Delta_{2}^{+}(\alpha)\left[\Delta_{3}^{+}(\alpha)+i C^{+} \Delta_{1}^{+}(\alpha)\right]=0, \\
\left\{\begin{array}{l}
\left|\Delta_{1}^{+}(\alpha)\right|^{2}-\left|\Delta_{2}^{+}(\alpha)\right|^{2}=0, \\
\left|\Delta_{3}^{+}(\alpha)+i C^{+} \Delta_{1}^{+}(\alpha)\right|^{2}-\left|\Delta_{4}^{+}(\alpha)-i C^{+} \Delta_{2}^{+}(\alpha)\right|^{2}=0,
\end{array}\right. \\
\overline{\Delta_{1}^{+}}(\alpha)\left[\begin{array}{l}
\left.\Delta_{3}^{+}(\alpha)+i C^{+} \Delta_{1}^{+}(\alpha)\right]-\overline{\Delta_{2}^{+}}(\alpha)\left[\Delta_{4}^{+}(\alpha)-i C^{+} \Delta_{2}^{+}(\alpha)\right]=0 .
\end{array}\right.
\end{gathered}
$$

Результаты применения параметрического подхода для отыскания вещественного главного показателя особенности решения прикладной задачи представлены ниже.

\section{4. Приложение к одной задаче теории упругости о трещине}

В качестве приложения используем разработанные подходы для определения показателей особенности решения СИУ, которое возникает в двумерной задаче теории упругости о прямолинейной трещинеразрезе единичной полудлины, один из концов которого лежит на границе полуплоскостей с различными упругими свойствами $[4,5]$. Для показателя особенности напряжений $\beta$ на этом конце разреза ключевыми параметрами являются угол $0<\gamma \leq \pi / 2$ между разрезом и границей раздела, а также отношение модулей сдвига материалов $\mu_{1} / \mu_{2}$ (индекс 1 соответствует полуплоскости с разрезом, коэффициент Пуассона $v_{1}=v_{2}=0,3$, плоская деформация). СИУ такой краевой задачи является одним из частных случаев (1), а обобщенное ядро $L_{g e n}(\xi, \eta)(3)$ оказывается равным нулю только в симметричном случае - при $\gamma=\pi / 2[4,5]$.

На рис. 1, $a$ представлены результаты расчета комплексного показателя особенности для сингулярной суперпозиции (19). Зависимость вещественной части показателя особенности $\beta$ от угла наклона $\gamma$ представлена сплошной кривой, а зависимость мнимой части штриховой (см. рис. 1). Как обсуждалось выше, для формы решения (19) показатели особенности предоставляются уравнением (22), распределение комплексных корней которого носит непрерывный харак- 
тер. В связи с последним обстоятельством в ходе решения уравнения (22) определялся корень с минимальной вещественной частью из этого непрерывного множества, и именно его зависимость от угла наклона при $\mu_{1} / \mu_{2}=5\left(\beta_{5 / 1}\right)$ и $\mu_{1} / \mu_{2}=1 / 5\left(\beta_{1 / 5}\right)$ представлена на рис. $1, a$. Для сравнения на этом же рисунке пунктирными кривыми представлены также зависимости $\left(\beta_{5 / 1}^{\prime}\right.$ и $\left.\beta_{1 / 5}^{\prime}\right)$ [5], которые соответствуют корню с минимальной вещественной частью из дискретного множества решений уравнений (15)-(17), т.е. для сингулярной формы (13).

Как видно, в данном случае комплексный главный показатель степенно-логарифмической особенности соответствует сингулярной суперпозиции (19) и предоставляется уравнением (22), свойственным также степенной асимптотике (см. (4) и (7)) (см. рис. 1, a). В то же время сложный характер функций в трансцендентных уравнениях (15)(17) и (22) не позволяет сделать однозначный вывод о том, какое из этих уравнений предоставляет главный показатель особенности в общем случае, поэтому анализ комплексной степенно-логарифмической асимптотики решения в каждом конкретном случае следует проводить для обеих форм (13) и (19). Следует отметить, что на правом конце диапазона $0<\gamma<\pi / 2$, где при $\gamma=\pi / 2 \quad L_{g e n}(\xi, \eta)=0$, и реализуется степенная особенность уравнения (7), представленные зависимости показателя степенно-логарифмической особенности, в том числе для уравнения (17) ( $\beta_{5 / 1}^{\prime}$ и $\beta_{1 / 5}^{\prime}$ ), стыкуются с известными вещественными значениями показателя степенной особенности напряжений для трещины, выходящей на интерфейс под прямым углом [1]. Аналогичная ситуация имеет место для представленных на рис. $1, a$ зависимостей при $\gamma$ приближающихся к нулю, где как вещественная, так и мнимая части показателя степенно-логарифмической особенности стремятся к аналитическому степенному решению для интерфейсной трещины $[6,7]$. Отметим также, что мнимые части главных показателей особенности в данном случае оказались очень близкими во всем диапазоне $0<\gamma<\pi / 2$ (см. рис. 1, $a$ ).

На рис. $1, \sigma$ представлены результаты расчетов при $\mu_{1} / \mu_{2}=5$ $\left(\beta_{5 / 1}\right)$ и $\mu_{1} / \mu_{2}=1 / 5\left(\beta_{1 / 5}\right)$ отыскиваемого в классе априори вещественных величин показателя особенности, полученные в рамках решения уравнения (9), единого для сингулярной суперпозиции (19) и классиче- 
ской степенной асимптотики (4). Отметим, что такого вида зависимости для степенной особенности были получены ранее в рамках прямого асимптотического анализа рассматриваемой краевой задачи $[20,21]$, а также на основе асимтотического анализа описывающего ее СИУ [4]. Результаты же данного исследования позволяют рассматривать эти зависимости в новом свете - во всем диапазоне углов наклона трещиныразреза $0<\gamma<\pi / 2$ реализуется более сильная степенно-логарифмическая особенность в составе сингулярной суперпозиции (19).
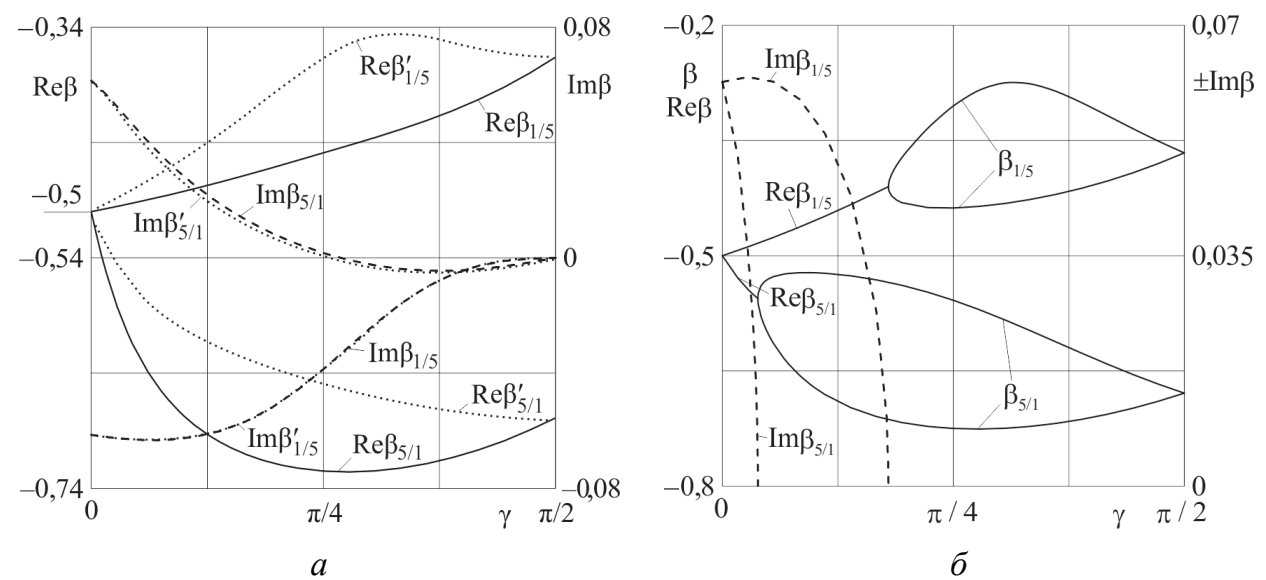

Рис. 1. Зависимость комплексного ( $a$ ) и вещественного (б) показателей особенности от угла наклона трещины к границе раздела

Как видно из рис. 1,6 , в данной прикладной задаче корни уравнения (9) составляют два вещественных, а при определенных параметрах задачи - два комплексно сопряженных значения. Уравнения относительно комплексного (7) и вещественного (9) показателей особенности, вообще говоря, различны, поэтому полученные в указанных диапазонах углов наклона трещины $\gamma$ комплексные значения показателя особенности (см. рис. 1, б) не удовлетворяют уравнению (7) (что подтверждается непосредственной подстановкой) и, следовательно, не являются допустимыми для СИУ и описываемой им краевой задачи теории упругости. В то же время непосредственное использование адекватных комплексных значений показателя особенности (см. рис. 1, a), полученных в рамках решения уравнения (7), для рассматриваемой задачи о трещине-разрезе приведет к появлению нефизических эффектов взаимопроникновения поверхностей трещины. 
На рис. 2 представлены результаты применения разработанного параметрического подхода к рассматриваемой прикладной задаче при $\mu_{1} / \mu_{2}=5$ (рис. $2, a$ ) и $\mu_{1} / \mu_{2}=1 / 5$ (рис. $2, \sigma$ ). Для удобства анализа на рис. 2 воспроизводятся зависимости рис. $1, \sigma$ для вещественных показателей особенности $\beta_{5 / 1}$ и $\beta_{1 / 5}$ от угла наклона трещины $\gamma$ в указанном его диапазоне, пунктирной кривой также показана вещественная часть комплексного показателя особенности. Зависимости вещественного показателя особенности $\beta_{C 1}=\beta_{C 1}(\gamma)$ (сплошные кривые) сингулярной суперпозиции (19) при условии (27) $(\alpha=-1 / 2)$ и вещественного параметра $C_{1}=C_{1}(\gamma)$ (штрихпунктирные кривые) получены в рамках решения комплексного уравнения (33), зависимости $\beta_{C 2}=\beta_{C 2}(\gamma)$ и $C_{2}=C_{2}(\gamma)$ - для уравнения (31), а вещественная система (32) не имеет корней в промежутке $-1<\beta<0$. Отметим, что для рассматриваемой задачи представленные корни уравнений (31) и (33) - единственные в промежутке $-1<\beta<0$.
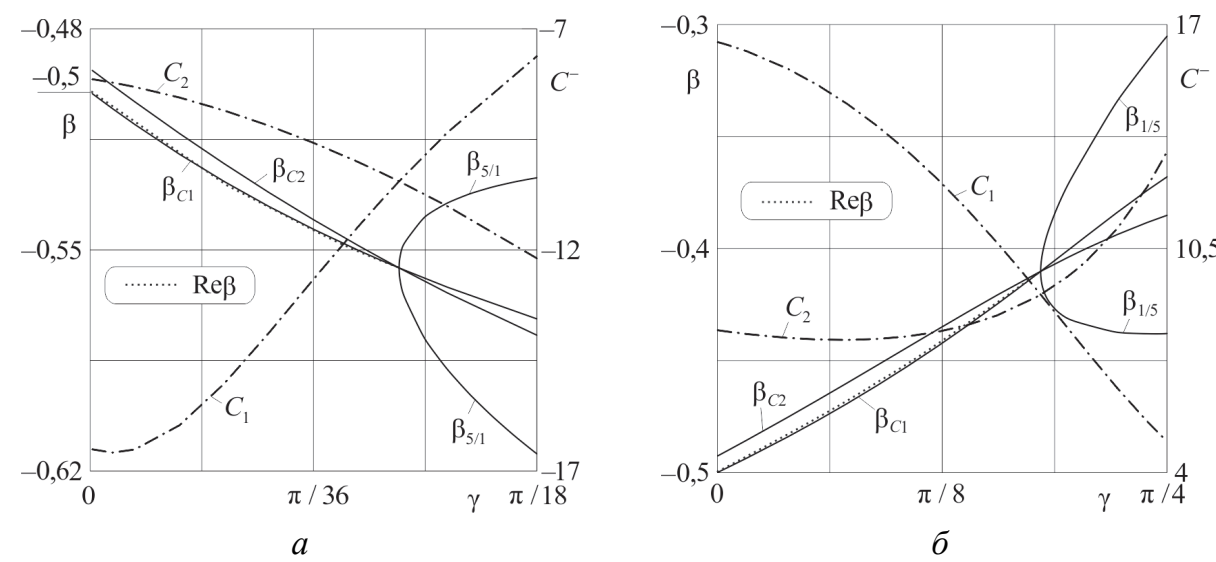

Рис. 2. Вещественные показатели особенности $\beta$ и параметры решения $C$ при отношении модулей сдвига $\mu_{1} / \mu_{2}=5$ (a) и $\mu_{1} / \mu_{2}=1 / 5$ (б)

Как видно из рис. 2, параметрический подход позволяет получить допустимые для СИУ (1) и описываемой им краевой задачи вещественные показатели особенности сингулярной суперпозиции (19) при соотношении (27) во всем диапазоне углов наклона трещины-разреза $\gamma$, где уравнение (7) имело только комплексные корни. Главный вещественный показатель особенности оказывается при этом очень близок 
к вещественной части комплексного показателя, а при $\gamma \rightarrow 0$ приближается к $-1 / 2$ (см. рис. 2), что соответствует вещественной части аналитического решения при $\gamma=0[6,7]$. Следует также отметить, что обе полученные в рамках параметрического подхода зависимости для вещественного показателя особенности естественным образом (т.е. без наложения дополнительных условий) сшиваются с вещественными показателями особенности непараметризованного подхода (см. рис. 2). Все эти обстоятельства свидетельствует в пользу разработанного параметрического подхода к построению неосциллирующей главной сингулярной асимптотики упругого поля.

\section{Заключение}

Таким образом, развитый ранее аппарат асимптотического анализа решений СИУ с обобщенными ядрами и сопряженными неизвестными позволил получить новые результаты для двумерных задач теории упругости о концентрации напряжений в однородных и кусочно-однородных областях с особыми точками. Показано, что наличие сопряженной неизвестной функции в характеристической части СИУ оказывает качественное влияние на сингулярный характер решения, главные особенности которого оказываются в такой ситуации не степенными, а степеннологарифмическими. С точки зрения двумерных задач теории упругости такая ситуация соответствует особой точке, в окрестности которой задача не разделяется на независимые нормальную и сдвиговую задачи, или, другими словами, компоненты перемещений не определяются независимо друг от друга. Поскольку такая ситуация характерна для очень широкого круга задач, многочисленные результаты, полученные ранее в рамках сингулярных решений теории упругости вблизи особых точек, требуют пересмотра и уточнения. В связи с этим результаты данного исследования имеют весьма важное значение для фундаментальных и прикладных задач теории концентрации напряжений на разрывах, контактной механики и механики разрушения.

Работа выполнена при финансовой поддержке Совета по грантам Президента РФ для ведущих научных школ (гранты НШ-4082.2014.1 и НШ-1275.2014.1). 


\section{Библиографический список}

1. Sinclair G.B. Stress singularities in classical elasticity-I: Removal, interpretation and analysis // Appl. Mech. Rev. - 2004. - Vol. 57. - No. 4. P. 251-297. doi: 10.1115/1.1762503

2. Sinclair G.B. Stress singularities in classical elasticity-II: Asymptotic identification // Appl. Mech. Rev. - 2004. - Vol. 57. - No. 5. - P. $385-$ 439. doi: $10.1115 / 1.1767846$

3. Paggi M., Carpintery A. On the stress singularities at multimaterial interfaces and related analogies with fluid dynamics and diffusion // Appl. Mech. Rev. - 2008. - Vol. 61. - P. 020801-1-22. doi: 10.1115/1.2885134

4. Андреев А.В. Метод определения комплексных особенностей степенного типа в решениях сингулярных интегральных уравнений с обобщенными ядрами и сопряженными неизвестными // Изв. РАН. MTT. - 2009. - № 5. - С. 42-58.

5. Андреев А.В. Степенно-логарифмические особенности решения одного класса сингулярных интегральных уравнений плоской теории упругости // Вычислительная механика сплошных сред. - 2014. T. 7, № 1. - C. 30-39. doi: 10.7242/1999-6691/2014.7.1.4

6. Erdogan F.E., Gupta G.D., Cook T.S. The numerical solutions of singular integral equations // Mechanics of fracture. Vol. 1. Methods of analysis and solutions of crack problems / Ed. G.C. Sih. - Noordhoff Intern. Publ., 1973. - P. 368-425.

7. Мусхелишвили Н.И. Сингулярные интегральные уравнения. М.: Наука, 1968. - 511 с.

8. Дудучава Р.В. Интегральные уравнения свертки с разрывными предсимволами, сингулярные интегральные уравнения с неподвижными особенностями и их приложения к задачам механики. - Тбилиси: Мецниереба, 1979. - 135 с.

9. Savruk M.P., Madenci E., Shkarayev S. Singular integral equations of the second kind with generalized Cauchy-type kernels and variable coefficients // Int. J. Numer. Meth. Eng. - 1999. - Vol. 45. - No. 10. P. 1457-1470. doi:10.1002/(SICI)1097-0207(19990810)45:10<1457: :AID-NME639>3.0.CO;2-P.

10. Williams M.L. Stress singularities resulting from various boundary conditions in angular corners of plates in extension // J. App. Mech. 1952. - Vol. 19. - No. 4. - P. 526-528. 
11. Каландия А.И. Замечания об особенности упругих решений вблизи углов // ПММ. - 1969. - Т. 33, Вып. 1. - С. 132-135.

12. Мусхелишвили Н.И. Некоторые основные задачи математической теории упругости. - М.: Наука, 1966. - 707 с.

13. Theocaris P.S. The order of singularity at a multiwedge corner of a composite Plate // Int. J. Eng. Sci. - 1974. - Vol. 12. - No. 2. - P. 107-120. doi: 10.1016/0020-7225(74)90011-1

14. Dempsey J. P. Power-logarithmic stress singularities at bi-material corners and interface cracks // J. Adhes. Sci. Technol. - 1995. - Vol. 9. No. 2. - P. 253-265. doi: 10.1163/156856195X01157

15. Саврук М.П. Двумерные задачи упругости для тел с трещинами. - Киев: Наук. думка, 1981. - 323 с.

16. Линьков А.М. Комплексный метод граничных интегральных уравнений теории упругости. - СПб.: Наука, 1999. - 382 с.

17. Михайлов С.Е. Сингулярность напряжений в окрестности ребра в составном неоднородном анизотропном теле и некоторые приложения к композитам // Изв. АН СССР. МТТ. - 1979. - №. 5. - С. $103-$ 110 .

18. Корепанова Т.О., Матвеенко В.П., Севодина Н.В. Численный анализ сингулярности напряжений в вершине конуса с негладкой боковой поверхностью // Вычислительная механика сплошных сред. 2010. - T. 3, № 3. - С. 68-76. doi: 10.7242/1999-6691/2010.3.3.28

19. Корепанова Т.О., Матвеенко В.П., Севодина Н.В. Численный анализ сингулярности напряжений в вершине пространственных пересекающихся трещин // Вычислительная механика сплошных сред. 2011. - T. 4, № 3. - C. 68-73. doi: 10.7242/1999-6691/2011.4.3.28

20. Fenner D.N. Stress singularities in composite materials with an arbitrarily oriented crack meeting an interface // Int. J. Fract. - 1976. Vol. 12. - No. 5. - P. 705-721. doi: 10.1007/BF00037917

21. Yong-Li W. Crack tip stress singularities in a bimaterial with an inclined interface // Int. J. Fract. - 1992. - Vol. 54. - No. 4. - P. R65-R72. doi: 10.1007/BF00035114

\section{References}

1. Sinclair G.B. Stress singularities in classical elasticity-I: Removal, interpretation, and analysis. Appl. Mech. Rev., 2004, vol. 57, no. 4, pp. 251297. doi: $10.1115 / 1.1762503$ 
2. Sinclair G.B. Stress singularities in classical elasticity-II: Asymptotic identification. Appl. Mech. Rev., 2004, vol. 57, no. 5, pp. 385-439. doi: $10.1115 / 1.1767846$

3. Paggi M., Carpintery A. On the stress singularities at multimaterial interfaces and related analogies with fluid dynamics and diffusion. Appl. Mech. Rev., 2008, vol. 61, pp. 020801-1-22. doi: 10.1115/1.2885134

4. Andreev A.V. Development of direct numerical integration methods for one-dimensional integro-differential equations in mechanics. $\mathrm{Me}$ chanics of Solids, 2009, vol. 42, iss. 2, pp. 209-222. doi: 10.3103/S0025654407020069

5. Andreev A.V. Stepenno-logarifmicheskie osobennosti resheniya odnogo klassa singuliarnykh integralnykh uravnenij ploskoj teorii uprugosti [The power-logarithmic singularities of solution for a class of singular integral equations arising in two-dimensional elasticity]. Computational continuum mechanics, 2014, vol. 7, no. 1, pp. 30-39. doi: 10.7242/19996691/2014.7.1.4

6. Erdogan F.E., Gupta G.D., Cook T.S. The numerical solutions of singular integral equations. Mechanics of fracture. Vol. 1. Methods of analysis and solutions of crack problems. Ed. G.C. Sih. Noordhoff Intern. Publ., 1973, pp. 368-425.

7. Muskhelishvili N.I. Singuliarnye integralnye uravneniya [Singular integral equations]. Moscow: Nauka, 1968. $511 \mathrm{~s}$.

8. Duduchava R.V. Integralnye uravneniya svertki s razryvnymi predsimvolami, singuliarnye integralnye uravneniya s nepodvizhnymi osobennostiami i ikh prilozheniya $\mathrm{k}$ zadacham mekhaniki [Convolution type integral equations with discontinuous presymbols, singular integral equations with fixed singularities, and their applications to problems in mechanics]. Tbilisi: Metsniereba, 1979. 135 s.

9. Savruk M.P., Madenci E., Shkarayev S. Singular integral equations of the second kind with generalized Cauchy-type kernels and variable coefficients. Int. J. Numer. Meth. Eng., 1999, vol. 45, no. 10, pp. 1457-1470. doi: 10.1002/(SICI)1097-0207(19990810)45:10<1457::AID-NME639>3.0.CO;2-P

10. Williams M.L. Stress singularities resulting from various boundary conditions in angular corners of plates in extension. J. App. Mech., 1952, vol. 19, no. 4, pp. 526-528.

11. Kalandia A.I. Zamechaniya ob osobennosti uprugikh reshenij vblizi uglov [Remarks on the singularity of elastic solutions near corners]. $P M M, 1969$, vol. 33, iss. 1, pp. 132-135. 
12. Muskhelishvili N.I. Nekotorye osnovnye zadachi matematicheskoj teorii uprugosti [Some basic problems of the mathematical theory of elasticity]. Moscow: Nauka, 1966. 707 p.

13. Theocaris P.S. The order of singularity at a multiwedge corner of a composite Plate. Int. J. Eng. Sci., 1974, vol. 12, no. 2, pp. 107-120. doi: 10.1016/0020-7225(74)90011-1

14. Dempsey J.P. Power-logarithmic stress singularities at bi-material corners and interface cracks. J. Adhes. Sci. Technol., 1995, vol. 9, no. 2, pp. 253-265. doi: 10.1163/156856195X01157

15. Savruk M.P. Dvumernye zadachi uprugosti dlia tel s treshchinami [Two-dimensional elasticity problems for bodies with cracks]. Kiev: Naukova dumka, 1981, 324 s.

16. Linkov A.M. Kompleksnyj metod granichnykh integralnykh uravnenij teorii uprugosti [Complex variable boundary integral equations of elasticity theory]. St. Petersburg: Nauka, 1999, $382 \mathrm{s.}$

17. Mikhailov S.E. Singuliarnost napriazhenij v okrestnosti rebra v sostavnom neodnorodnom anizotropnom tele i nekotorye prilozheniya $\mathrm{k}$ kompozitam [Stress singularity near edge in the compound inhomogeneous anisotropic body and some applications for composites]. Izvestiya Akademii nauk SSSR. MTT, 1979, no. 5, pp. 103-110.

18. Korepanova T.O., Matveenko V.P., Sevodina N.V. Chislennyj analiz singuliarnosti napriazhenij $\mathrm{v}$ vershine konusa $\mathrm{s}$ negladkoj bokovoj poverkhnostju [Numerical analysis of stress singularities at the apex of a cone with unsmooth lateral surface]. Computational continuum mechanics, 2010, vol. 3, no. 3, pp. 68-76. doi: 10.7242/1999-6691/2010.3.3.28

19. Korepanova T.O., Matveenko V.P., Sevodina N.V. Chislennyj analiz singuliarnosti napriazhenij $\mathrm{v}$ vershine prostranstvennykh peresekayushchikhsya treshchin [Numerical analysis of stress singularities at the tip of intersecting 3D wedge-shaped cracks]. Computational continuum mechanics, 2011, vol. 4, no. 3, pp. 68-73. doi: 10.7242/1999-6691/2011.4.3.28

20. Fenner D.N. Stress singularities in composite materials with an arbitrarily oriented crack meeting an interface. Int. J. Fract., 1976, vol. 12, no. 5, pp. 705-721. doi: 10.1007/BF00037917

21. Yong-Li W. Crack tip stress singularities in a bimaterial with an inclined interface. Int. J. Fract., 1992, vol. 54, no. 4, pp. R65-R72. doi: 10.1007/BF00035114 


\section{Об авторе}

Андреев Андрей Вячеславович (Москва, Россия) - кандидат физико-математических наук, старший научный сотрудник Объединенного института высоких температур РАН (125412, г. Москва, ул. Ижорская, 13. стр. 2, e-mail: andrey.andreev@inbox.ru).

\section{About the author}

Andrey V. Andreev (Moscow, Russian Federation) - Ph.D. in Physical and Mathematical Sciences, Senior Scientist of the Joint Institute for High Temperatures of the Russian Academy of Sciences (13, Bd. 2, Izhorskaya str., 125412, Moscow, Russian Federation, e-mail: andrey.andreev@inbox.ru).

Получено 12.02.2014

Просьба ссылаться на эту статью в русскоязычных источниках следующим образом:

Андреев А.В. Суперпозиция степенно-логарифмических и степенных сингулярных решений в двумерных задачах теории упругости // Вестник Пермского национального исследовательского политехнического университета. Механика. - 2013. № $1 .-$ C. 5-30.

Please cite this article in English as:

Andreev A.V. Superposition of power-logarithmic and power singular solutions in two-dimensional elasticity problems. PNRPU Mechanics Bulletin. 2013. No. 1. P. 5-30. 